\title{
Guía de práctica clínica de síndrome metabólico
}

Antonio González-Chávez ${ }^{1 *}$, Joan Erick Gómez-Miranda², Sandra Elizondo-Argueta ${ }^{3}$, María del Pilar Rangel-Mejía ${ }^{4}$ y Martín de Jesús Sánchez-Zúñiga ${ }^{5}$

${ }^{1}$ Servicio de Medicina Interna, Hospital General de México Dr. Eduardo Liceaga, Secretaría de Salud; ${ }^{2}$ Dirección General de Guías de Práctica Clínica, Centro Nacional de Excelencia Tecnológica en Salud, Secretaría de Salud; ${ }^{3}$ División de Proyectos Especiales en Salud, Instituto Mexicano del Seguro Social; ${ }^{4}$ Servicio de Medicina Interna, Hospital General Regional n. ${ }^{\circ} 1$ Dr. Carlos MacGregor Sánchez Navarro, Instituto Mexicano del Seguro Social; ${ }^{5}$ Servicio de Terapia Intensiva, Instituto Nacional de Rehabilitación Dr. Luis Guillermo Ibarra Ibarra, Secretaría de Salud. Ciudad de México, México

\section{RESUMEN}

Introducción: El síndrome metabólico (SM) es uno de los principales factores de riesgo para desarrollar diabetes tipo 2 y enfermedad cardiovascular, su diagnóstico temprano y oportuno es una herramienta para establecer medidas de prevención hacia complicaciones crónicas y en consecuencia evitar una mala calidad de vida del paciente. Metodología: Se realizó una revisión sistemática de la literatura con base en seis preguntas clínicas estructuradas que incluían los desenlaces más importantes para la población y tema de estudio, con la finalidad de encontrar evidencia disponible y establecer recomendaciones clave. Resultados: Se obtuvieron 163 documentos; solo 27 fueron incluidos para formar parte del cuerpo de evidencia, la cual de manera global fue considerada como de moderada a baja calidad metodológica. Con respecto a la seguridad a largo plazo de la intervención fue considera la incorporación de evidencia indirecta, dada la falta de información en la población de interés. Conclusio-
ABSTRACT

Introduction: Metabolic Syndrome (MS) is one of the principal risk factors for type 2 diabetes and cardiovascular disease; its early and timely diagnosis is a tool to establish prevention actions and avoid chronic complications and a poor quality of life. Methodology: A systematic review was made based on six structured clinical questions that included the most important outcomes for the population and issue in study, the aim was to find available evidence and establish key recommendations. Results: 163 documents were obtained; only 27 of them were included into the part of the evidence, all of them were considered as with moderate to low methodological quality. Regarding the long-term safety of the intervention, it was considered the incorporation of indirect evidence, given the lack of information in the population of interest. Conclusions: 19 recommendations (12 strong and 7 weak) and 8 points of good practice were issued to be a reference on the relevance of the prevention, diagnosis and
Correspondencia:

*Antonio González-Chávez

E-mail: antglez51dyahoo.com.mx
Fecha de recepción: 04-03-2019

Fecha de aceptación: 24-06-2019 DOI: 10.24875/ALAD.19000381 
nes: Se emitieron 19 recomendaciones (12 fuertes y 7 débiles) y 8 puntos de buena práctica que permiten ser un referente sobre la relevancia de la prevención, diagnóstico y tratamiento del SM como medida de prevención de enfermedades no transmisibles, sin embargo aún se requiere mayor evidencia sobre esta enfermedad.

Palabras clave: Guía de práctica clínica. Síndrome metabólico. Diagnóstico. Tratamiento timely treatment of MS as a preventive action for noncommunicable diseases; however, greater specific evidence about MS is necessary. (Rev ALAD. 2019;9:179-200) Corresponding author: Antonio González-Chávez, antglez51@yahoo.com.mx

Key words: Clinical Practice Guidelines. Metabolic Syndrome. Diagnosis. Treatment.

\section{INTRODUCCIÓN}

El síndrome metabólico (SM) es un conjunto de factores de riesgo que pueden ser categorizados en cinco grupos: dislipidemia aterogénica, presión arterial elevada, disglicemia, estado protrombótico y estado proinflamatorio. La suma de estos factores incrementa de dos a cinco veces el riesgo de desarrollar diabetes y el riesgo de enfermedad cardiovascular en una a dos veces, además del desarrollo de otras comorbilidades como obesidad, hipertensión y dislipidemia, por lo que su diagnóstico temprano brindará una gran oportunidad de establecer medidas terapéuticas con la finalidad de prevenir estos desenlaces, donde la evidencia muestra que invertir en la prevención de enfermedades no transmisibles es costo-efectivo'.

Uno de los principales problemas no solo para su diagnóstico sino también al tratar de identificar su prevalencia, es la dificultad de integrar las diferentes definiciones de SM (Tabla 1). Las principalmente utilizadas han sido las de la Organización Mundial de la Salud (OMS), la del National Cholesterol Education Program-Third Adult Treatment Panel (ATP III) y la de la Federación Internacional de Diabetes (FID).
Aunque cada definición posee características comunes a las otras, los factores que las diferencian hacen que los parámetros para integrar la información sean complicados en términos de aplicabilidad, uniformidad y valores predictivos positivos ${ }^{2,3}$.

Los estudios poblacionales de prevalencia del SM muestran cifras muy variables dependiendo del grupo étnico, zona geográfica, ambiente sociocultural $y$, por supuesto, de la definición empleada para su determinación, sin embargo, aun así se observa que su prevalencia sigue en aumento ${ }^{4}$. En el estudio CARMELA, al aplicar la definición del ATP III se encontraron importantes variaciones en la prevalencia del SM en diferentes ciudades de Latinoamérica. La prevalencia más alta en personas con edades entre 25 y 64 años se encontró en Ciudad de México (27\%), seguida por Barquisimeto (26\%), Santiago de Chile (21\%), Bogotá (20\%), Lima (18\%), Buenos Aires (17\%) y finalmente Quito (14\%) 5 . En una revisión sistemática de 10 estudios hechos en adultos en Brasil el promedio ponderado de prevalencia del SM fue del 29.6\% (rango: 14.9-65.3\%), empleando diferentes definiciones, siendo la más alta del $65.3 \%$ en población indígena y la más baja en población rural, del 14,9\% . Otra revisión sistemática de prevalencia de SM en 
TABla 1. Criterios diagnósticos más utilizados de síndrome metabólico

\begin{tabular}{|c|c|c|c|c|c|}
\hline Criterio diagnóstico & $\begin{array}{l}\text { Obesidad } \\
\text { (abdominal) }\end{array}$ & Triglicéridos, mg/dl & C-HDL, mg/dl & $\begin{array}{l}\text { Presión arterial, } \\
\mathrm{mmHg}\end{array}$ & Nivel glucosa mg/dl \\
\hline $\begin{array}{l}\text { IDF, } 2005 \\
\text { Diagnóstico obesidad } \\
\text { abdominal }+\geq 2 \text { componentes }\end{array}$ & $\begin{array}{l}\geq 90 \mathrm{H}^{*} \\
\geq 80 \mathrm{M}^{*}\end{array}$ & $\geq 150$ & $\begin{array}{c}<40 \mathrm{H} \\
<50 \mathrm{M} \\
\text { Tratamiento }\end{array}$ & $\begin{array}{l}\geq 130 / 850 \\
\text { tratamiento } \\
\text { antihipertensivo }\end{array}$ & $\begin{array}{l}\geq 100 \text { o } \\
\text { diagnóstico de DM2 }\end{array}$ \\
\hline $\begin{array}{l}\text { Update ATP III, } 2005 \\
\text { Diagnóstico } \geq 3 \text { componentes }\end{array}$ & $\begin{array}{l}>102 \mathrm{H} \\
>88 \mathrm{M}\end{array}$ & $\geq 150$ & $\begin{array}{c}<40 \mathrm{H} \\
<50 \mathrm{M} \\
\text { Tratamiento }\end{array}$ & $\begin{array}{l}\geq 130 / 850 \\
\quad \text { tratamiento } \\
\text { antihipertensivo }\end{array}$ & $\begin{array}{l}\text { Glucosa ayuno } \geq 110 \\
\text { inicial o } \\
\text { diagnóstico de DM2 }\end{array}$ \\
\hline $\begin{array}{l}\text { ALAD, } 2007 \\
\text { Diagnóstico obesidad } \\
\text { abdominal }+\geq 2 \text { componentes }\end{array}$ & $\begin{array}{l}\geq 94 \mathrm{H}^{\ddagger} \\
\geq 88 \mathrm{M}^{\ddagger}\end{array}$ & $\begin{array}{l}\geq 150 \text { o tratamiento } \\
\text { para triglicéridos }\end{array}$ & $\begin{array}{c}<40 \mathrm{H} \\
<50 \mathrm{M} \\
\text { Tratamiento }\end{array}$ & $\begin{array}{l}\geq 130 / 850 \\
\text { tratamiento } \\
\text { antihipertensivo }\end{array}$ & GAA, ITG o DM \\
\hline $\begin{array}{l}\text { Criterios armonizados, } 2009 \\
\text { Diagnóstico } \geq 3 \text { componentes }\end{array}$ & $\begin{array}{l}\geq 94 \mathrm{H}^{\dagger} \\
\geq 88 \mathrm{M}^{\dagger}\end{array}$ & $\begin{array}{l}\geq 1500 \\
\text { tratamiento } \\
\text { para triglicéridos }\end{array}$ & $\begin{array}{l}<40 \mathrm{H} \\
<50 \mathrm{M}\end{array}$ & $\begin{array}{l}\geq 130 / 850 \\
\text { tratamiento } \\
\text { antihipertensivo }\end{array}$ & $\begin{array}{l}\geq 100 \text { o } \\
\text { diagnóstico de } \mathrm{DM} 2 \\
\text { o tratamiento } \mathrm{DM} 2\end{array}$ \\
\hline
\end{tabular}

H: hombres; M: mujeres; GAA: glucosa en ayuno alterada; ITG: intolerancia a la glucosa; IDF: International Diabetes Federation; ATP III: Adult Treatment Panel III; DM2: diabetes tipo 2; C-HDL: high densitiy lipoproteins cholesterol.

*Valores para asiáticos.

tValores para latinoamericanos.

‡Valores para latinoamericanos (el de mujeres se homologó con ATP III).

Latinoamérica incluyó 11 estudios en adultos (18 a 65 años) y utilizando la definición del ATP III observó un promedio ponderado del 24.9\% (rango: 18.8$43.3 \%$ ), la prevalencia fue un poco más alta en mujeres ( 25.3 vs. $23.2 \%$ en hombres) y en personas mayores de 50 años?.

El SM debe reconocerse como un problema mayor y creciente para la salud pública y de un gran desafío clínico, no solo por las implicaciones en la salud, también porque su origen está definido a raíz de factores externos modificables pero que conllevan grandes retos a vencer por su relación con temas de políticas públicas, como aspectos de urbanización, acceso a alimentos, contaminación y estrés, entre otros.

En la práctica clínica la detección del SM no se realiza de manera sistematizada y cuando por azar esta se realiza, el seguimiento de los pacientes es nulo, no se considera la figura de una persona con factores de riesgo para desarrollo de SM y, más aún, por muchos el SM aún no se considera como una enfermedad. La evidencia ha demostrado que un abordaje sistemático de detección oportuna y el establecimiento de programas efectivos tanto no farmacológicos como farmacológicos son estrategias efectivas (y mejor aún: costo-efectivas) para disminuir la carga de la enfermedad metabólica y sus complicaciones.

A través de esta guía de práctica clínica (GPC) se busca emitir recomendaciones basadas en un análisis de la evidencia científica, así como de la experiencia de los profesionales de la salud expertos en la materia, para que los médicos puedan realizar intervenciones en salud con la finalidad de brindar una atención de calidad a su paciente con SM. Su enfoque y alcances se limitan a pacientes mayores de 18 años con factores de riesgo para el desarrollo de SM y/o que cumplan criterios diagnósticos.

\section{METODOLOGÍA}

Se generaron un total de seis preguntas, por medio de un formato estructurado y de manera priorizada, 
las cuales fueron elegidas por consenso con el fin de disminuir la subjetividad del proceso ( $\mathrm{P}$ : población; I: intervención; E: exposición; C: comparación; O: descenlace):

1. ¿Cuáles son los factores de riesgo con mayor asociación para desarrollar SM en adultos?

P: población adulta; E: sedentarismo (tiempo), aumento de la circunferencia abdominal, aumento del índice de masa corporal (IMC), patrones occidentales de alimentación, peso al nacimiento, antecedente de SM familiar; C: control, dieta mediterránea; O: riesgo de SM.

2. ¿Cuáles son las estrategias de prevención primaria más eficaces para disminuir el riesgo de desarrollo de SM en adultos?

P: adultos mayores de 18 años; I: modificaciones del estilo de vida (MEV) (terapia conductual, dieta, ejercicio, dieta más ejercicio); C: control, comparación directa entre cada intervención; O: riesgo de SM con base en modificaciones de componentes individuales y en conjunto.

3. ¿Cuáles son los padecimientos con mayor asociación para incrementar el riesgo metabólico en los adultos?

P: pacientes adultos mayores de 18; E: diabetes gestacional, enfermedad mental mayor, síndrome de ovario poliquístico (SOP); C: control; O: riesgo de SM.

4. ¿Cuáles son los criterios clínicos con mayor utilidad para calcular la probabilidad diagnóstica del SM en los pacientes adultos?

P: adultos mayores de 18; E: ATP III, FID, Asociación Estadounidense del Corazón/Instituto Nacional del Corazón, los Pulmones y la Sangre
(AHA/NHLBI), Asociación Americana de Endocrinólogos Clínicos (AACE), Programa Nacional de Educación en Colesterol (NCEP-R); C: criterios de Organización Mundial de la Salud (OMS); O: sensibilidad, especificidad, porcentaje de valor predictivo positivo (\%VPP), porcentaje de valor predictivo negativo (\%VPN), nivel de concordancia en la aplicación (medido por valor de kappa).

5. ¿Cuáles son las estrategias de tratamiento no farmacológico con mayor eficacia para el SM en el primer nivel en los adultos?

$\mathrm{P}$ : pacientes adultos con diagnóstico de SM; I: terapia conductual (dieta + ejercicio + terapia psicológica, grupos de auto ayuda), dieta con restricción calórica, dieta con restricción calórica + ejercicio, ejercicio; C: control, tratamiento farmacológico, tratamiento farmacológico + ejercicio; O: remisión del SM, modificación en los componentes individuales del SM.

6. ¿Cuáles son las estrategias de tratamiento farmacológico con mayor eficacia para el SM en el primer nivel en los adultos?

P: adultos mayores de 18 años con diagnóstico de SM; I: tratamiento farmacológico; C: control, manejo médico, terapia conductual (dieta + ejercicio + terapia psicológica, grupos de auto ayuda), dieta con restricción calórica, dieta con restricción calórica + ejercicio, ejercicio; O: remisión del SM, modificación en los componentes individuales del SM.

\section{Búsqueda sistemática de la evidencia y su evaluación crítica}

Al ser formuladas las preguntas clínicas, se definió la estrategia búsqueda de la GPC y revisiones sistemáticas nacionales e internacionales disponibles 


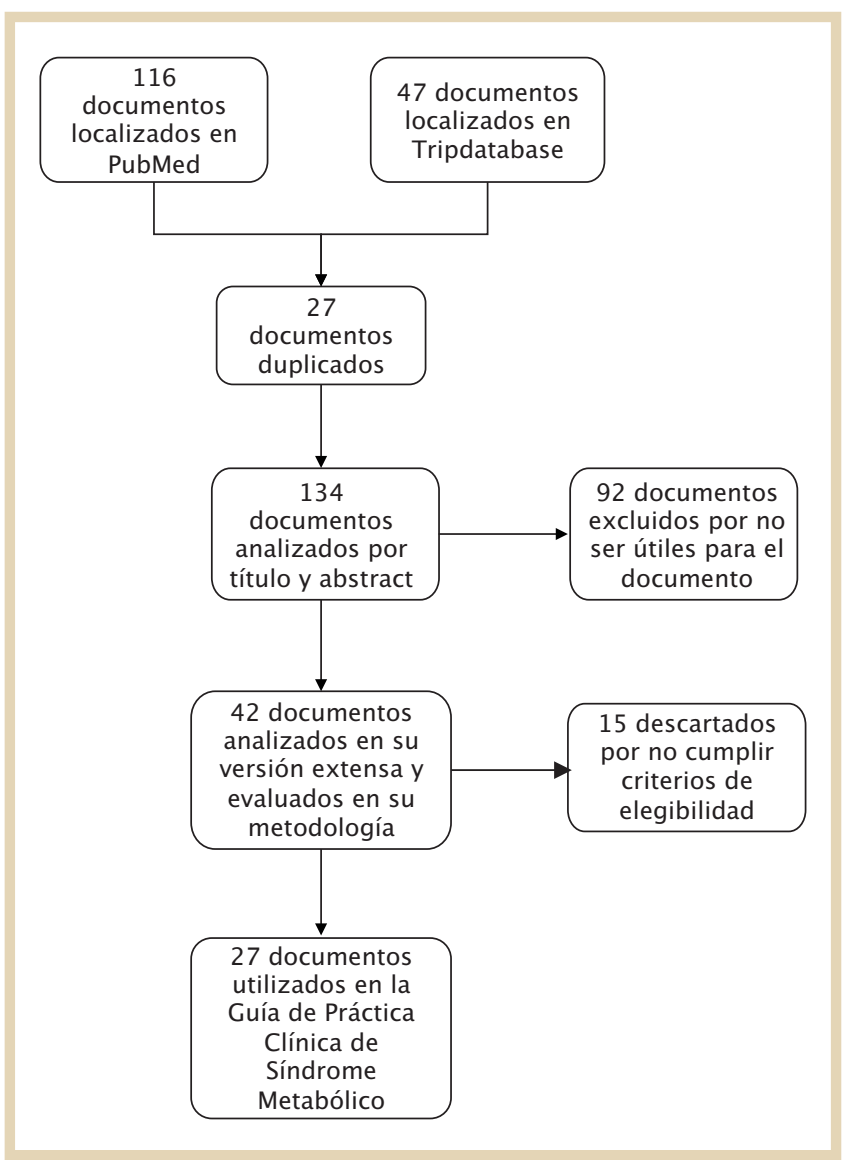

Figura 1. Diagrama de flujo de selección de documentos.

relacionadas con el tema de interés, dentro de un margen temporal de no más de cinco años anteriores al año 2018 (Fig. 1). Dos miembros del grupo desarrollador seleccionaron los documentos potencialmente útiles mediante título y resumen. Las publicaciones seleccionadas fueron evaluadas con base en su validez metodológica con ayuda de dos integrantes del grupo desarrollador y de manera independiente mediante el sistema GRADE (Grading of Recommendations, Assessment, Development and Evaluation).

Para la formulación de recomendaciones se utilizaron los datos contenidos en las tablas de evidencia, aunado a la experiencia clínica del grupo desarrollador. Para cada recomendación se realizó un consenso estructurado. Las recomendaciones se realizaron de manera que fuesen de fácil entendimiento y concisas para facilitar el proceso de implementación. Para el caso en donde la evidencia no exista o sea insuficiente se llevó a cabo un consenso para la generación de puntos de buena práctica.

\section{EVIDENCIAS Y RECOMENDACIONES}

\section{Factores de riesgo con mayor asociación para desarrollar síndrome metabólico en adultos}

\section{SEDENTARISMO \\ Recomendación fuerte}

Se deben reducir las conductas sedentarias para disminuir el riesgo de SM. Las personas con más tiempo de comportamiento sedentario tienen mayores probabilidades de desarrollar SM.

Para adultos de 18 a 64 años recomendamos realizar actividad física que incluya actividades físicas recreativas o de tiempo libre, disminuir el transporte en vehículos automotores y realizar transporte activo (p. ej., caminar o andar en bicicleta), ocupacional (es decir, trabajo), tareas domésticas, juegos, deportes o ejercicio planificado, aplicándolas a las actividades de la vida diaria.

Para los adultos de más de 65 años recomendamos la actividad física que incluya actividades físicas recreativas o de tiempo libre, transporte activo (p. ej., caminar o andar en bicicleta), ocupacional (si la persona todavía está ocupada en el trabajo), tareas domésticas, juegos, deportes o ejercicios planificados, en el contexto de las actividades diarias, familiares y comunitarias, con el fin de mejorar la salud cardiorrespiratoria y muscular, la salud ósea y la capacidad funcional, y reducir el riesgo de enfer- 
TABLA 2. ¿Cuál es la asociación del sedentarismo como factor de riesgo en el SM en adultos comparado con individuos sin conductas sedentarias?

\begin{tabular}{|c|c|c|c|}
\hline \multicolumn{4}{|c|}{$\begin{array}{l}\text { Pregunta: } \\
\text { P: pacientes adultos; E: conductas sedentarias; C: sin conductas sedentarias; O: riesgo de síndrome metabólico. }\end{array}$} \\
\hline \multicolumn{4}{|c|}{ Paciente o población: adultos mayores de 18} \\
\hline Desenlaces & $\begin{array}{l}\text { N. }{ }^{\circ} \text { de participantes } \\
\text { (n. }{ }^{\circ} \text { de estudios) }\end{array}$ & $\begin{array}{l}\text { Certeza de la evidencia } \\
\text { (GRADE) }\end{array}$ & $\begin{array}{l}\text { Efecto relativo } \\
\text { IC } 95 \%)\end{array}$ \\
\hline SM en hombres sedentarios & $\begin{array}{l}2,217 \text { casos } 6,340 \text { controles } \\
(5 \text { estudios observacionales) }\end{array}$ & $\begin{array}{l}\oplus \oplus \oplus \bigcirc \\
\text { Moderado }\end{array}$ & $\begin{array}{l}\text { OR: } 1.54 \\
(1.28 \text { a } 1.58)\end{array}$ \\
\hline SM en mujeres sedentarias & $\begin{array}{l}2,053 \text { casos } 7,984 \text { controles } \\
(6 \text { estudios observacionales) }\end{array}$ & $\begin{array}{l}\oplus \oplus \oplus \bigcirc \\
\text { Moderado* }\end{array}$ & $\begin{array}{l}\text { OR: } 2.07 \\
(1.70 \text { a } 2.52)\end{array}$ \\
\hline SM ajustado por tipo de actividad física & $\begin{array}{l}5,104 \text { casos } 13,585 \text { controles } \\
\text { ( } 8 \text { estudios observacionales) }\end{array}$ & $\begin{array}{l}\oplus \oplus \oplus \bigcirc \\
\text { Moderado* }\end{array}$ & $\begin{array}{l}\text { OR: } 1.73 \\
(1.54 \text { a } 1.97)\end{array}$ \\
\hline
\end{tabular}

El riesgo en el grupo de intervención (y su intervalo de confianza del 95\%) se basa en el riesgo asumido en el grupo de comparación y en el efecto relativo de la intervención (y su intervalo de confianza del $95 \%$ ).

*Rangos de edad variables, diferentes criterios utilizados para diagnóstico de SM.

GRADE: Grading of Recommendations, Assessment, Development and Evaluation; IC: intervalo de confianza; OR: razón de momios; SM: síndrome metabólico.

Adaptada de Edwardson, et al., $2012^{8}$.

medades no transmisibles, depresión y deterioro cognitivo.

\section{Nivel de evidencia moderado (Tabla 2)}

Se calcularon las OR (odds ratio, razón de momios) para el SM con relación al estilo de vida sedentario y los resultados en salud. Los datos se calcularon utilizando un modelo de efectos aleatorios en el metaanálisis. Se identificaron diez estudios transversales ( $n=21,393$ participantes). El resultado principal estableció que a mayor tiempo de vida sedentaria aumenta de manera significativa la probabilidad de padecer SM hasta en un 73\% (OR: 1.73; intervalo de confianza [IC] 95\%: 1.55-1.94; $\mathrm{p}<0.0001$ ). No hubo diferencias para los subgrupos de sexo, método de medición del comportamiento sedentario, definición del SM, calidad del estudio o ingreso del país. No hubo evidencia de heterogeneidad estadística ( $I^{2}: 0.0 \% ; p=0.61$ ) o sesgo de publicación (prueba de Eggers, t: $1.05 ; p=0.32)^{8}$.

\section{Alimentación}

\section{Recomendación fuerte}

Se debe llevar un patrón de alimentación saludable que evite o disminuya de manera importante la ingesta de patrones occidentales poco saludables, ya que disminuye de significativamente el riesgo para desarrollar SM. Se debe diseñar un plan de alimentación con la ayuda de un nutriólogo, que favorezca la pérdida de peso en los pacientes con obesidad o sobrepeso.

\section{Nivel de evidencia moderado}

En un metaanálisis sobre patrones de dieta y SM, la OR agrupada (IC del 95\%) para el SM en comparación de la categoría más alta a la más baja de los patrones dietéticos occidentales en los estudios transversales fue de 1.28 (IC 95\%: 1.17-1.40; I²: 72\%). El riesgo relativo (RR) agrupado (IC del 95\%) para el SM en los estudios de cohortes fue de 0.96 (IC 95\%: 0.53-1.73; I²: 62.6\%). 
Las fuentes potenciales de heterogeneidad produjeron cambios menores y diferencias no significativas en los resultados. En los análisis de sensibilidad, la exclusión de los estudios individuales no modificó sustancialmente las estimaciones agrupadas en la OR para SM, que varió de 1.25 a 1.31 en los estudios transversales. Los resultados de los estudios transversales mostraron que un patrón de alimentación saludable está asociado con un menor prevalencia de SM, mientras que un estilo occidental de alimentación (frutas [frutas en conserva], granos refinados [pasta, arroz, pan blanco y cereal para el desayuno], lácteos [leche entera, queso], proteínas [carne roja, carne procesada], aceites y grasas [mantequilla, margarina, salsas grasas, ensalada alta en grasa aderezos], bebidas alcohólicas [cerveza, licores], refrescos, café, dulces [helados, pasteles, galletas, chocolate] y comida rápida [pizza, refrigerios, papas fritas]) se asocia con un mayor riesgo de SM. Se requiere evidencia adicional de estudios prospectivos para confirmar la asociación entre los patrones dietéticos y el SM99.

\section{Perímetro de circunferencia abdominal}

\section{Recomendación fuerte}

Se debe realizar la medición del perímetro de cintura y mantenerlo dentro de los parámetros establecidos para las diferentes poblaciones, dada su importante asociación con otros componentes del $\mathrm{SM}$; la disminución de este se relaciona con mejoría de los niveles de glucosa y los lípidos séricos, cambios que son directamente proporcionales a la disminución del perímetro de cintura.

\section{Nivel de evidencia moderado}

En un estudio cuyo objetivo fue evaluar el impacto de los cambios en la circunferencia abdominal sobre la presión arterial, los lípidos y la glucemia, se incluyeron 430 pacientes obesos inscritos en un programa de control de peso que involucró terapia conductual y manejo médico intensivo durante dos años. Los resultados obtenidos fueron de los participantes que completaron un seguimiento de seis meses $y$ hasta dos años. Sus edades promedio eran $49 \pm$ 9 años de edad, el 56\% eran mujeres y el $85 \%$ de raza blanca. El IMC inicial fue de $41 \pm 6 \mathrm{~kg} / \mathrm{m}^{2}$ y la línea base para la circunferencia abdominal fue $120 \pm 14 \mathrm{~cm}$. En un lapso de seis meses y tras someterse a rutinas de ejercicio intenso y estrategias dietéticas, el IMC disminuyó en $6 \pm 3 \mathrm{~kg} / \mathrm{m}^{2}$ y la circunferencia abdominal disminuyó en promedio $14 \pm 9 \mathrm{~cm}$. El cálculo del cambio en la circunferencia abdominal se definió como la circunferencia abdominal a los seis meses o dos años menos la medida inicial de la circunferencia dividido entre la línea base de la circunferencia abdominal. La presión arterial sistólica (PAS) disminuyó en $8 \mathrm{mmHg}$ para el tercil de participantes con la disminución relativa más grande en la circunferencia abdominal y en $2 \mathrm{mmHg}$ para aquellos con una disminución más modesta $(p=0.025)$. Patrones similares fueron observados en las modificaciones de los niveles de colesterol total ( -29 vs. $-12 \mathrm{mg} / \mathrm{dl} ; \mathrm{p}=0.017$ ), colesterol LDL (low-density lipoprotein) (-19 vs. $-4 \mathrm{mg} / \mathrm{dl}$; $\mathrm{p}=0.033)$, y hemoglobina glucosilada ( -1.2 vs. $-0.3 \%$; $\mathrm{p}=0.006)$. A los dos años, el IMC disminuyó en $5 \pm$ $4 \mathrm{~kg} / \mathrm{m}^{2}$ y la circunferencia abdominal en $11 \pm 11 \mathrm{~cm}$ y se observaron cambios similares en cuanto a la mejoría del resto de los componentes del SM. De los seis meses a los dos años, las diferencias en las mejoras en los niveles séricos de lípidos y de glucosa fueron mayores e independientes del sexo del paciente ${ }^{10}$.

\section{ÍNDICE DE MASA CORPORAL}

\section{Recomendación fuerte}

Se debe determinar en todos los pacientes el IMC, puesto que el sobrepeso es un factor constante 
inicial del SM en la mayor parte de los pacientes. Estas mediciones deben realizarse desde la infancia, ya que influyen directamente en la presencia de obesidad y diabetes en la vida adulta.

\section{Nivel de evidencia moderado}

Todos los estudios incluidos en la revisión sistemática se publicaron entre 2008 y 2014, y los participantes de este estudio solo eran asiáticos o europeos. Se incluyeron 12 resultados de cinco estudios en el metaanálisis. El sobrepeso u obesidad en la primera infancia se asoció con un mayor riesgo de SM en adultos en comparación con los controles. Cuando se confirmó en cada grupo de edad (al nacimiento, 0-2 y 2-6 años), hubo una diferencia estadísticamente significativa antes y después de la edad de 2 años. Como resultado de la metarregresión, cuando la edad de los niños aumentó, el tamaño del efecto del SM del adulto para el sobrepeso u obesidad también aumentó. Los resultados confirman que el SM tiene efectos a largo plazo desde etapas tempranas de la vida e indican que se necesita una intervención temprana para el sobrepeso u obesidad. En los adultos no se ha establecido evidencia de adecuada calidad metodológica para establecer la presencia de sobrepeso como un factor común para presentar SM, si se considera que aunque poco común, existen personas con sobrepeso sin presencia de SM, por lo que deben estar asociados otros factores en el desarrollo de alteraciones bioquímicas ${ }^{11-13}$.

\section{Peso al nacimiento}

\section{Recomendación débil}

La evidencia demuestra que un alto peso al nacer predispone a un sobrepeso posterior y así se asocia al desarrollo de SM. Se recomienda prevenir condiciones que se asocien con desarrollo de sobrepeso del producto, como la diabetes gestacional.

\section{Nivel de evidencia baja}

Se encontró que el peso al nacer, de 2,500 g, fue seguido por un menor riesgo de sobrepeso (OR: 0.67; IC 95\%: 0.59-0.76). El alto peso al nacer, de 4,000 g, se asoció con un mayor riesgo de sobrepeso (OR:1.66; IC 95\%: 1.55-1.77). Los resultados no cambiaron significativamente al considerar el peso normal al nacer (2,500-4,000 g) como categoría de referencia (OR: 0.73, IC 95\%: 0.63-0.84; OR: 1.60, IC 95\%: 1.451.77, respectivamente). Los análisis de subgrupos e influencia revelaron que no hay presencia de sesgo de confusión. Las estimaciones ajustadas indican una duplicación del riesgo de sobrepeso a largo plazo es alta en comparación con sujetos con peso normal al nacer (OR: 1.96; IC 95\%: 1.43-2.67) ${ }^{14}$.

\section{ANTECEDENTE FAMILIAR DE SM}

\section{Recomendación débil}

El antecedente de SM familiar se asocia con la presencia de factores de riesgo de SM, lo que permite sugerir la necesidad de evaluación diagnóstica temprana en pacientes jóvenes con este antecedente.

\section{Nivel de evidencia bajo}

En un estudio con adultos jóvenes sanos se identificó la presencia de SM familiar y en comparación con los participantes control, se observó un colesterol total significativamente más alto (5.46 vs. $4.69 \mathrm{mmol} / \mathrm{l}$; $p<0.030$ ), colesterol LDL (3.28 vs. $2.90 \mathrm{mmol} / \mathrm{l}$; $\mathrm{p}<0.032$ ) y niveles de colesterol HDL (high density 
lipoproteins) (3.74 vs. $3.25 \mathrm{mmol} / \mathrm{l} ; \mathrm{p}<0.016$ ), además de niveles de glucosa en ayuno menores (4.51 vs. $4.81 \mathrm{mmol} / \mathrm{l} ; \mathrm{p}$ <0.042). Se detectó una correlación positiva entre glucosa en ayuno y niveles de insulina ( $r: 0.28$; $p$ < 0.015) en los participantes con SM familiar. Mayor PAS media durante el día (121.5 vs. $113.3 \mathrm{mmHg} ; \mathrm{p}<0.035)$ y presión arterial diastólica (PAD) diurna media (79.0 vs. 74.5 mmHg; $\mathrm{p}<0.045$ ), y se observaron valores medios de PAD durante la noche (64.0 vs. 59.5 mmHg; $p$ < 0.019) en el grupo pacientes con SM. Más del $50 \%$ de los participantes con SM familiar tenían exceso de peso o un trastorno lipídico, lo que puede indicar un mayor riesgo de enfermedad cardiovascular y la necesidad de una evaluación ambulatoria regular de las concentraciones de lípidos en suero en jóvenes con antecedentes familiares de $S M^{15}$.

\section{Estrategias de prevención primaria más eficaces para disminuir el riesgo de desarrollo de síndrome metabólico en adultos}

\section{Punto de buena práctica}

Las MEV representan una serie de estrategias que se aplican en la prevención de varias enfermedades no transmisibles y que se encuentran asociadas al SM (obesidad, diabetes); al no existir evidencia específica, se recomienda la aplicación de estas medidas en prevención del SM, como dietas con restricción calórica para la reducción de peso, bajas en sodio, bajas en grasas saturadas, e incremento de la actividad física principalmente.

\section{Punto de buena práctica}

Las MEV que se implementen como medidas de prevención para SM deberán siempre estar acordes con las necesidades y situación del paciente, y deberán respetar aspectos culturales, sociales, económicos, educativos y religiosos, entre otros, y así lograr como tal el cambio conductual.

\section{Alimentación}

\section{Recomendación fuerte}

Se recomienda limitar la ingesta de carbohidratos simples como una de las estrategias iniciales de manejo en los pacientes con sobrepeso y factores de riesgo para presentar SM, o en aquellos que presentan alteraciones bioquímicas asociadas a este. Aunado a esto, se debe instruir a los pacientes para generar cambios en el estilo de vida que incluyan además rutinas de ejercicio de acuerdo con sus capacidades físicas individuales.

\section{Nivel de evidencia moderado}

En un metaanálisis que incluyó 2,788 participantes, y que comparó dieta baja en carbohidratos (< $45 \%$ de energía procedente de carbohidratos) y dieta baja en grasas (< $30 \%$ de energía procedente de grasas) sugiere que las dietas bajas en carbohidratos son tan efectivas como las dietas bajas en grasa para reducir el peso y mejorar los factores de riesgo metabólico, entre ellos el colesterol LDL y trigicéridos ${ }^{16}$.

\section{EJERCICIO}

\section{Recomendación fuerte}

Se debe recomendar al paciente la realización de ejercicio como medida terapéutica para la prevención de factores de riesgo cardiometabólico, el cual deberá ser individualizado. 


\section{Nivel de evidencia moderado}

Un metaanálisis evaluó el efecto del ejercicio en factores de riesgo cardiovascular en pacientes con SM. Incluyó 206 participantes con edad promedio de 52 años (rango entre 46 y 64 años), en quienes la frecuencia de entrenamiento varió entre $2 \mathrm{y}$ 5 sesiones semanales (media de 3 ) con promedio de 47.5 minutos por sesión y con actividades como caminar, jogging y bicicleta, con intensidades de moderadas a altas. Se observó una reducción del perímetro de cintura de -3.4 (IC 95\%: -4.9 a -1.8 ) cm, presión sistólica/diastólica de -7.1 (IC 95\%: -9.03 a -5.2)/-5.2 (IC 95\%: -6.2 a -4.1) mmHg y un incremento en colesterol HDL de 0.06 (IC 95\%: 0.03-0.09). Sin embargo estos resultados no pueden ser extrapolados a pacientes con edades diferentes dadas sus características ${ }^{17}$.

\section{Padecimientos con mayor asociación para aumentar el riesgo metabólico en los adultos de manera significativa}

\section{DIABETES GESTACIONAL}

\section{Recomendación fuerte}

Las pacientes con diabetes gestacional deben mantenerse en vigilancia estricta posterior al parto en relación a los componentes individuales relacionados al SM, dado el riesgo elevado de desarrollarlo.

\section{Nivel de evidencia moderado}

Las mujeres con antecedentes de DMG (diabetes gestacional) tenían un riesgo significativamente mayor de desarrollar SM que las que tuvieron un embarazo normal (OR: 3.96; IC 95\%: 2.99-5.26), pero los resultados presentan una heterogeneidad significativa ( $\left.{ }^{2}: 52.6 \%\right)$. El efecto permaneció robusto (OR: 4.54; IC 95\%: 3.78-5.46) en el subgrupo de pacientes caucásicos, pero no se encontró asociación en los asiáticos (OR: 1.28; IC 95\%: 0.64-2.56). Además, las madres con un IMC más alto en el grupo de DMG tuvieron un mayor riesgo de desarrollar SM que las del grupo con solo elevación en el IMC (IMC mayor en el grupo con DMG, OR: 5.39, IC 95\%: 4.47-6.5; grupo con IMC equivalente, OR: 2.53 ; IC 95\%: 1.88-3.41) ${ }^{18}$.

\section{ENFERMEDADES MENTALES Y USO DE ANTIPSICÓTICOS}

\section{Recomendación débil}

Se recomienda realizar estrategias de cribado y de prevención para SM en pacientes con enfermedades mentales severas, principalmente cuando se asocian con tratamiento a base de antipsicóticos.

\section{Nivel de evidencia moderado}

La prevalencia combinada de SM en personas con enfermedad mental severa calculada en esta revisión sistemática fue del 32.6\% (IC 95\%: 30.8-34.4\%; $N=198 ; n=52,678)$. Los metaanálisis de riesgo relativo establecieron que no hubo diferencias significativas en la prevalencia de SM en los estudios comparando directamente la esquizofrenia con el trastorno bipolar, y en aquellos que comparan directamente el trastorno bipolar vs. el trastorno depresivo mayor. Las personas tratadas con todos los medicamentos antipsicóticos individuales tuvieron un riesgo de SM significativamente $(p<0.001)$ más alto en comparación con los participantes que inician con la ingesta de antipsicóticos. En comparación con los controles de población general combinados, las personas con enfermedad mental grave tenían un riesgo 
significativamente mayor de SM (RR: 1.58; IC 95\%: 1.35-1.86; $p<0.001$ ) y todos sus componentes, a excepción de la hipertensión $(p=0.07)^{19}$.

\section{SÍNDROME DE OVARIO POLIQUÍSTICO}

\section{Recomendación débil}

Las pacientes con diagnóstico de síndrome de ovario poliquístico (SOP) deben ser sometidas a cribado para descartar la presencia de SM.

\section{Nivel de evidencia moderada}

En una revisión sistemática y metaanálisis que incluyó 18,529 mujeres con SOP y 15,891 controles sanas, se observó que las pacientes con SOP, independientemente de la edad e IMC, tenían un riesgo elevado de SM comparado con controles sanas (OR: 2.5; IC 95\%: $2.0-3.2)^{20}$.

\section{Criterios clínicos con mayor utilidad para el diagnóstico del síndrome metabólico en adultos}

\section{Punto de buena práctica}

Debido a la ausencia de una definición única, y la existencia de varios criterios diagnósticos relacionados que han surgido por diferentes entidades internacionales, se sugiere que el médico utilice aquella que sea de más fácil acceso, ante la necesidad de realizar pruebas diagnósticas para su implementación (como son la de la FID y el ATP III), y que se adapte a las características de la población a la que será aplicada, dado que muchas de estas definiciones no han sido validadas para población latinoamericana.

\section{Recomendación débil}

Se recomienda utilizar los criterios de la FID o del ATP III para realizar cribado de SM.

\section{Nivel de evidencia baja}

Con el fin de establecer cuál es la serie de criterios con mayor utilidad para diagnosticar SM, se realizó una búsqueda de la literatura. Se localizaron tres estudios observacionales en los cuales se analiza tal cuestionamiento, en dos de ellos se analiza la prevalencia del SM utilizando los diferentes criterios diagnósticos en población con alto riesgo, dado que se involucran personas con diabetes tipo 2 (DM2) y pacientes con trastornos mentales, los cuales se encuentran bajo tratamiento farmacológico. En otro de los estudios se describe la utilidad de siete criterios para diagnosticar SM en una población con riesgo promedio. La calidad de la evidencia es de baja a muy baja y no existe evidencia directa de adecuada calidad proveniente de población latinoamericana. En la tabla 3 se pueden observar los resultados de la comparación de la capacidad diagnóstica para el SM entre las diferentes directrices y grado según los criterios, donde el nivel de acuerdo parece ser en general deficiente, aunque los criterios de la FID mostraron un nivel de acuerdo justo con los criterios de la OMS; además, los criterios de la FID demostraron ser superiores a otros (ATP III, NCEP-R, AACE, OMS), ya que presentaron un VPP del $96.3 \%$. Por lo tanto, los criterios de la FID se recomiendan para el cribado del SM en personas con DM2 debido a su facilidad de aplicación y su nivel de acuerdo con los criterios de la OMS (tomados como gold standard) es el mejor en comparación con los otros tres criterios ${ }^{21}$. La capacidad diagnóstica de los criterios de la FID son seguidos muy de cerca. 
TABLA 3. ¿Cuáles son los criterios diagnósticos con mayor utilidad en el SM en pacientes adultos?

\begin{tabular}{|c|c|c|c|c|c|c|c|c|c|}
\hline \multicolumn{10}{|c|}{$\begin{array}{l}\text { P: población adulta; E: criterios Federación Internacional de Diabetes (FID), National Cholesterol Education Program-Third Adult Treatment } \\
\text { Panel (ATP III NCEP), National Cholesterol Education Program (NCEP.R), American Association of Clinical Endocrinologists (AACE); } \\
\text { C: criterios de la OMS; O: sensibilidad, especificidad, valores predictivos positivos, valores predictivos negativos, nivel de concordancia. }\end{array}$} \\
\hline \multirow[t]{2}{*}{ Criterio } & \multicolumn{2}{|c|}{$\begin{array}{l}\text { Criterio de } \\
\text { referencia } \\
\text { OMS }\end{array}$} & \multirow[t]{2}{*}{ Sensibilidad } & \multirow[t]{2}{*}{ Especificidad } & \multirow[t]{2}{*}{ VPP $\%$} & \multirow[t]{2}{*}{ VPN $\%$} & \multirow[t]{2}{*}{$\begin{array}{l}\text { Índice de } \\
\text { concordancia } \\
\text { kappa }\end{array}$} & \multirow[t]{2}{*}{$\begin{array}{l}\text { Nivel de } \\
\text { acuerdo }\end{array}$} & \multirow[t]{2}{*}{$\begin{array}{l}\text { Certeza de } \\
\text { la evidencia } \\
\text { (GRADE) }\end{array}$} \\
\hline & SM & Sin SM & & & & & & & \\
\hline \multicolumn{10}{|l|}{ FID } \\
\hline Negativos & 77 & 3 & \multirow[b]{2}{*}{71.2} & \multirow[b]{2}{*}{81.3} & \multirow[b]{2}{*}{96.1} & \multirow[b]{2}{*}{29.5} & \multirow[b]{2}{*}{0.30} & \multirow{2}{*}{ Malo } & \multirow[b]{2}{*}{ Bajo } \\
\hline Positivos & 31 & 13 & & & & & & & \\
\hline \multicolumn{9}{|l|}{ ATP III } & \\
\hline Negativos & 66 & 3 & \multirow{2}{*}{61.1} & \multirow[b]{2}{*}{81.3} & \multirow[b]{2}{*}{95.7} & \multirow[b]{2}{*}{23.6} & \multirow[b]{2}{*}{0.21} & \multirow[b]{2}{*}{ Malo } & \multirow[b]{2}{*}{ Bajo } \\
\hline Positivos & 42 & 13 & & & & & & & \\
\hline \multicolumn{9}{|l|}{ NCEP-R } & \\
\hline Positivos & 73 & 3 & \multirow{2}{*}{67.6} & \multirow{2}{*}{81.3} & \multirow{2}{*}{96.4} & \multirow{2}{*}{27.1} & \multirow{2}{*}{0.26} & \multirow{2}{*}{ Malo } & \multirow[b]{2}{*}{ Bajo } \\
\hline Negativos & 35 & 13 & & & & & & & \\
\hline \multicolumn{9}{|l|}{ AACE } & \\
\hline Positivos & 28 & 0 & \multirow[b]{2}{*}{25.9} & & & & & & \\
\hline Negativos & 80 & 16 & & 100 & 100 & 16.6 & 0.08 & Leve & Bajo \\
\hline
\end{tabular}

SM: síndrome metabólico; VPP: valor predictivo positivo; VPN: valor predictivo negativo; OMS: Organización Mundial de la Salud; FID: Federación Internacional de Diabetes; ATP III NCEP: National Cholesterol Education Program-Third Adult Treatment Panel; NCEP-R: National Cholesterol Education Program; AACE: American Association of Clinical Endocrinologists; GRADE: Grading of Recommendations, Assessment, Development and Evaluation.

Adaptada de Onesi, et al., 2014²1.

En otro estudio, se calculó la prevalencia de SM utilizando los criterios de la FID y los criterios del ATP III. Los criterios adaptados del ATP III y los de la FID para SM identificaron los mismos casos (el 29\% de todos los pacientes con esquizofrenia). Entre los criterios de la FID, la hipertrigliceridemia tenía la máxima sensibilidad, identificando correctamente al $77.8 \%$ de los pacientes. Los niveles bajos del colesterol HDL fue el criterio más específico, con un $95 \%$ de especificidad, que equivale a una razón de verosimilitud positiva de 9.78. Por lo tanto, los criterios de la FID y del ATP III pueden ser igualmente útiles para identificar el $\mathrm{SM}^{22}$.

En un último estudio seleccionado, se compara el rendimiento de siete criterios diagnósticos diferentes del SM con respecto a su prevalencia, las carac- terísticas de los sujetos con un diagnóstico positivo y la capacidad de identificar correctamente a las personas con alto riesgo cardiovascular (nivel de RCV calculado) o con signos de inflamación sistémica o daño orgánico temprano. Los criterios de diagnóstico propuestos por la OMS, European Group for the Study of Insulin Resistance (EGIR), el ATP III, la AACE, la FID y la AHA/NHLBI se aplicaron a una población de 933 hombres de 59.5 años de edad (3381 años) que asistieron al examen entre el 2002 y el 2004 en el Olivetti Heart Study. La prevalencia del SM varió del 8.6\% (AACE) al 44.5\% (FID). Entre los pacientes con $\mathrm{SM}$, la resistencia a la insulina varió del 94.8\% (EGIR) al 49.2\% (FID), mientras que la DM2 (excluida por EGIR y AACE) calificó el 59.9\% por la OMS y del 22 al $24 \%$ por ATP III, FID o AHA/NHBLI. 
Según la mayoría de los criterios, los sujetos con SM tenían un mayor RCV calculado que los sujetos sin SM; pero en general, la capacidad para identificar correctamente a los individuos con alto RCV se vio atenuada por una sensibilidad limitada (máximo $60 \%$ ). Bajando el límite para la adiposidad abdominal (circunferencia de cintura $\leq 94 \mathrm{~cm}$ por FID) no mejoró el rendimiento en este aspecto, pero identificó un mayor número de individuos con microalbuminuria (56\%) y proteína C reactiva elevada $(53 \%)^{23}$.

\section{Estrategias de tratamiento no farmacológico con mayor eficacia para el SM en adultos en el primer nivel de atención ${ }^{24-30}$}

\section{Punto de buena práctica}

Hay dos posibles enfoques terapéuticos para el SM. Una estrategia es identificar cada factor de riesgo por separado y tratar a cada factor sin relación con su agrupamiento con otros factores de riesgo. La estrategia alternativa es enfocarse en todos o en múltiples factores de riesgo con terapias únicas.

Se recomienda que el médico priorice las acciones terapéuticas a seguir en forma individualizada, tomando en cuenta las características de salud-enfermedad del paciente, pero también la presencia de factores externos que sean condicionantes del logro de metas y apego terapéutico, como entorno social, educativo, económico y cultural, entre otros.

\section{Punto de buena práctica}

El médico debe establecer como prioridad en el paciente MEV, las cuales se puede implementar mediante estrategias educativas, ya sea individuales o grupales, con el objetivo no solo de lograr las metas establecidas, sino de mantener el apego a estas y que estas se conviertan en costumbres y puedan ser transmitidas a su familia y comunidad, con base en la evidencia que existe sobre su beneficio al ser aplicable a otras enfermedades no transmisibles.

\section{MODIFICACIONES DEL ESTILO DE VIDA}

\section{Recomendación fuerte}

A los individuos con riesgo de desarrollar o con diagnóstico de SM se les debe recomendar adherirse a un plan dietético y de actividad física personalizado como parte de las MEV, cuyo objetivo será crear un cambio de comportamiento y de hábitos de la vida diaria de manera duradera.

\section{Nivel de evidencia moderado (Tabla 4)}

La modificación de los estilos de vida fue efectiva en la resolución de SM y en la reducción de la severidad de alteraciones relacionadas (glucosa sanguínea en ayunas, circunferencia de cintura, PAS, PAD y triglicéridos). Los tipos de modificaciones en los estilos de vida en los estudios seleccionados variaron hasta cierto punto, y se puede diferenciar entre aquellos que usan intervención dietética solo y aquellos que usan dieta más ejercicio. Once intervenciones en ocho ensayos controlados y aleatorizados se utilizaron para realizar los metaanálisis. La proporción relativa de pacientes con SM resuelto en el grupo de intervención fue de aproximadamente 2.0 veces mayor en el grupo de intervención (IC 95\%: 1.5-2.7) en comparación con el grupo de control (7 intervenciones, $n=2,839$ ). Las intervenciones de modificaciones en los estilos de vida redujeron significativamente ( 5 intervenciones, $n=748$ ) los valores medios para la presión sistólica en -6.4 (IC 95\%: -9.7 a -3.2) mmHg, la presión diastólica en -3.3 
TABLA 4. ¿Cuál es la eficacia de las modificaciones en el estilo de vida en pacientes con SM en comparación con no realizar modificaciones en el estilo de vida sobre los componentes individuales del SM en adultos?

P: población adulta con SM; I: modificaciones en el estilo de vida (tiempo medio de duración 12 meses incluidas dieta mediterránea, dieta DASH, educación dietética, planes de ejercicio); C: no realizar modificaciones en el estilo de vida; O: modificaciones en los niveles séricos de glucosa en ayuno, circunferencia abdominal, presión arterial sistólica, presión arterial diastólica, triglicéridos, colesterol HDL (resolución del SM).

\begin{tabular}{|c|c|c|c|c|c|c|c|c|c|c|c|c|}
\hline \multicolumn{7}{|c|}{ Evaluación de la certeza } & \multicolumn{2}{|c|}{ N. ${ }^{\circ}$ de pacientes } & \multicolumn{2}{|c|}{ Efecto } & \multirow[t]{2}{*}{ Certeza } & \multirow[t]{2}{*}{ Importancia } \\
\hline $\begin{array}{l}\text { N. }{ }^{\circ} \text { de } \\
\text { estudios }\end{array}$ & $\begin{array}{l}\text { Diseño de } \\
\text { estudio }\end{array}$ & $\begin{array}{l}\text { Riesgo } \\
\text { de sesgo }\end{array}$ & Inconsistencia & $\begin{array}{l}\text { Evidencia } \\
\text { indirecta }\end{array}$ & Imprecisión & Otras consideraciones & $\begin{array}{c}\text { Modificaciones } \\
\text { en el estilo } \\
\text { de vida }\end{array}$ & $\begin{array}{c}\text { No realizar } \\
\text { modificaciones }\end{array}$ & $\begin{array}{l}\text { Relativo } \\
\text { (IC 95\%) }\end{array}$ & $\begin{array}{l}\text { Absoluto } \\
\text { (IC 95\%) }\end{array}$ & & \\
\hline \multicolumn{13}{|c|}{ Glucosa en ayuno (7 intervenciones) (evaluado con: mg/dl) } \\
\hline 8 & $\begin{array}{l}\text { Ensayos } \\
\text { aleatorios }\end{array}$ & Serio* & No es serio & No es serio & No es serio & $\begin{array}{l}\text { Se sospechaba } \\
\text { fuertemente sesgo de } \\
\text { publicación. Todos los } \\
\text { posibles factores de } \\
\text { confusión residuales } \\
\text { podrían reducir el efecto } \\
\text { demostrado }{ }^{+}\end{array}$ & 1,400 & 1,439 & - & $\begin{array}{c}\text { MD } 11.5 \text { menor } \\
\text { (22.4 menor a } \\
0.6 \text { menor) }\end{array}$ & $\begin{array}{l}\oplus \oplus \oplus \bigcirc \\
\text { Moderado }\end{array}$ & Crítico \\
\hline
\end{tabular}

Circunferencia abdominal (6 intervenciones) (evaluado con: $\mathrm{cm}$ )

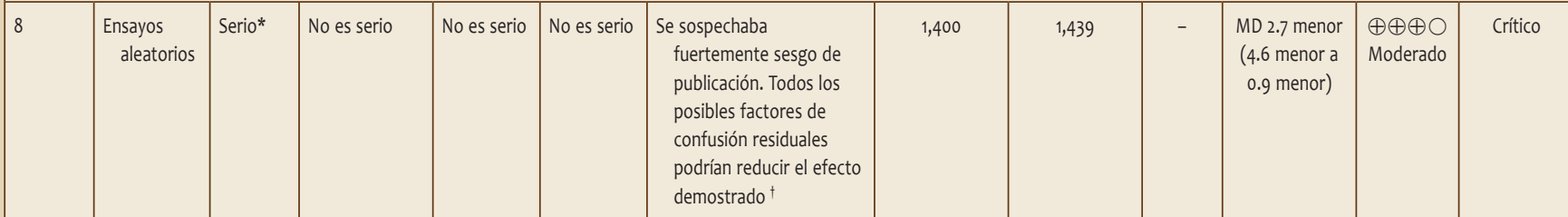

Presión arterial sistólica (evaluado con: $\mathrm{mmHg}$ )

\begin{tabular}{|c|c|c|c|c|c|c|c|c|c|c|c|c|}
\hline 8 & $\begin{array}{l}\text { Ensayos } \\
\text { aleatorios }\end{array}$ & Serio* & No es serio & No es serio & No es serio & $\begin{array}{l}\text { Se sospechaba } \\
\text { fuertemente sesgo de } \\
\text { publicación. Todos los } \\
\text { posibles factores de } \\
\text { confusión residuales } \\
\text { podrían reducir el efecto } \\
\text { demostrado }{ }^{+}\end{array}$ & 1,400 & 1,439 & - & $\begin{array}{c}\text { MD } 6.4 \text { menor } \\
\text { (9.7 menor a } \\
3.2 \text { menor) }\end{array}$ & $\begin{array}{l}\oplus \oplus \oplus \bigcirc \\
\text { Moderado }\end{array}$ & Crítico \\
\hline
\end{tabular}

Presión arterial diastólica (evaluado con: $\mathrm{mmHg}$ )

\begin{tabular}{|c|c|c|c|c|c|c|c|c|c|c|c|c|}
\hline 8 & $\begin{array}{l}\text { Ensayos } \\
\text { aleatorios }\end{array}$ & Serio* & No es serio & No es serio & No es serio & $\begin{array}{l}\text { Se sospechaba } \\
\text { fuertemente sesgo de } \\
\text { publicación. Todos los } \\
\text { posibles factores de } \\
\text { confusión residuales } \\
\text { podrían reducir el efecto } \\
\text { demostrado }\end{array}$ & 1,400 & 1,439 & - & $\begin{array}{c}\text { MD } 3.3 \text { menor } \\
\text { (5.2 menor a } \\
3.4 \text { menor) }\end{array}$ & $\begin{array}{l}\oplus \oplus \oplus \bigcirc \\
\text { Moderado }\end{array}$ & Crítico \\
\hline
\end{tabular}

Triglicéridos (evaluado con: $\mathrm{mg} / \mathrm{dl}$ )

\begin{tabular}{|c|c|c|c|c|c|c|c|c|c|c|c|c|}
\hline 8 & $\begin{array}{l}\text { Ensayos } \\
\text { aleatorios }\end{array}$ & Serio* & No es serio & No es serio & No es serio & $\begin{array}{l}\text { Se sospechaba } \\
\text { fuertemente sesgo de } \\
\text { publicación. Todos los } \\
\text { posibles factores de } \\
\text { confusión residuales } \\
\text { podrían reducir el efecto } \\
\text { demostrado }^{\dagger}\end{array}$ & 1,400 & 1,439 & - & $\begin{array}{c}\text { MD } 12 \text { menor } \\
(22.2 \text { menor a } \\
11.7 \text { menor })\end{array}$ & $\begin{array}{l}\oplus \oplus \oplus \bigcirc \\
\text { Moderado }\end{array}$ & Crítico \\
\hline \multicolumn{13}{|c|}{ Colesterol HDL (evaluado con: mg/dl) } \\
\hline 8 & $\begin{array}{l}\text { Ensayos } \\
\text { aleatorios }\end{array}$ & Serio* & No es serio & No es serio & No es serio & $\begin{array}{l}\text { Se sospechaba } \\
\text { fuertemente sesgo de } \\
\text { publicación. Todos los } \\
\text { posibles factores de } \\
\text { confusión residuales } \\
\text { podrían reducir el efecto } \\
\text { demostrado }^{+}\end{array}$ & 1,400 & 1,439 & - & $\begin{array}{c}\text { MD } 1.3 \\
\text { más alto. } \\
\text { ( } 0.6 \text { menor } \\
\text { a } 3.1 \text { más alto.) }\end{array}$ & $\begin{array}{l}\oplus \oplus \oplus \bigcirc \\
\text { Moderado }\end{array}$ & Crítico \\
\hline
\end{tabular}

*El riesgo de sesgo fue calificado como claro para la mayor parte de los estudios.

†Solo se localizaron estudios en inglés, por lo cual se puede ver una sobreestimación del efecto en un $2 \%$.

DASH: Dietary Approaches a Stop Hypertension. IC: intervalo de confianza; MD: diferencia media; SM: síndrome metabólico; HDL: high densitiy lipoproteins.

Adaptada de Yamaoka, et al., $2012^{25}$. 


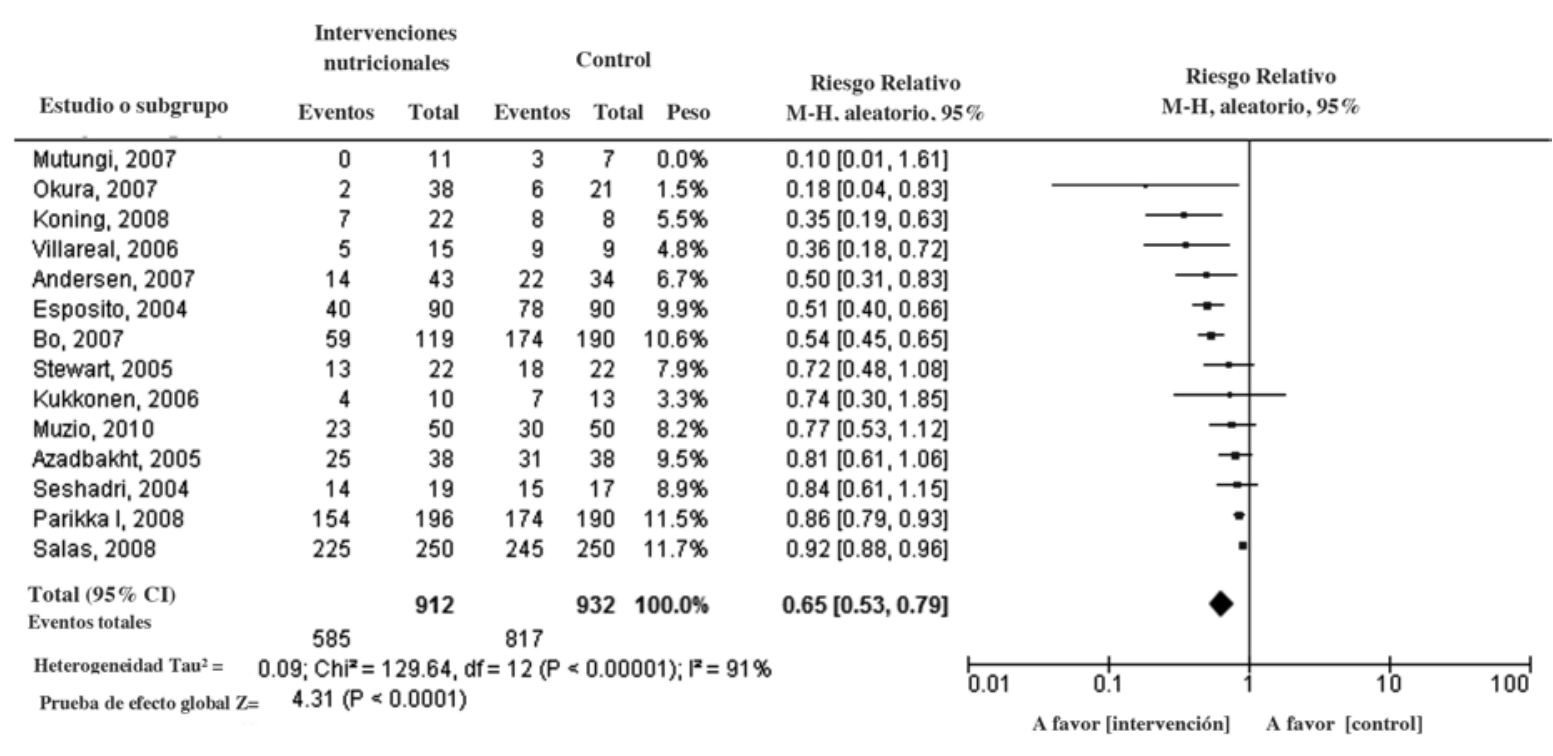

Fıgura 2. ¿Cuáles son las intervenciones nutricionales con mayor eficacia para el tratamiento del SM en adultos?.

Forest plot de estrategias nutricionales vs. control en pacientes con SM, análisis por efectos aleatorios.

Se omite el metaanálisis Mutungi, 2007 por tener muy poca población dentro de su estudio, el cual no modifica significativamente el resultado final.

DASH: Dietary Approaches a Stop Hypertension; GRADE: Grading of Recommendations, Assessment,

Development and Evaluation; SM: síndrome metabólico; IC: intervalo de confianza (adaptada de Moraes, et al., 201126).

P: pacientes adultos con SM; I: intervenciones nutricionales (dieta normocalórica, dieta baja en calorías,

dieta mediterránea, dieta DASH (Dietary Approaches a Stop Hypertension, dieta baja en calorías alta en

carbohidratos); C: manejo convencional (dieta hipocalórica); 0: disminución de riesgo de SM (resolución del SM).

Certeza en la evidencia: baja (GRADE).

(IC 95\%: -5.2 a -1.4) mmHg, los triglicéridos en -12.0 (IC 95\%: -22.2 a -1.7) $\mathrm{mg} / \mathrm{dl}$, la circunferencia abdominal en -2.7 (IC 95\%: -4.6 a -0.9$) \mathrm{cm}$ y la glucemia en ayunas en -11.5 (IC 95\%: -22.4 a -0.6 ) $\mathrm{mg} / \mathrm{dl}$ (5 intervenciones), pero las reducciones fueron no significativas para HDL (1.3, IC 95\%: -0.6 a $3.1 \mathrm{mg} / \mathrm{dl})^{17}$.

\section{Alimentación}

\section{Recomendación débil}

Actualmente no existe un consenso sobre las recomendaciones dietéticas más apropiadas, así como las que involucran MEV. La mayor parte de las recomendaciones sugieren dietas con restricción calórica, antiaterogénica y baja en grasas.
Se recomienda que los pacientes con SM tengan una dieta alta en grasas insaturadas, en carbohidratos complejos y no refinados, y en fibra (10-25 g/día), y baja en grasas saturadas y en azúcares y sodio (restringida de 2-5 g al día), ya que el sodio está asociado con la hipertensión. Además, se recomienda evitar el consumo de bebidas alcohólicas y bebidas con edulcorantes artificiales, así como evitar el consumo de tabaco.

\section{Nivel de evidencia baja (Fig. 2)}

Independientemente del tipo de intervención nutricional, el efecto observado en el metaanálisis es un efecto protector sobre el riesgo de presentar SM para todas las propuestas de tratamiento nutricional analizadas. 
Existe una gran heterogeneidad en los diferentes estudios incluidos y poca calidad metodológica, sin embargo no existe actualmente en la literatura evidencia de adecuada calidad y que compare exclusivamente intervenciones nutricionales, dado que en todos los estudios además de cambios en la dieta se incluye la actividad física dentro del plan de manejo.

\section{ACTIVIDAD FÍSICA}

\section{Recomendación débil}

Se recomienda el ejercicio aeróbico realizando diariamente un mínimo de 30 minutos de leve a moderado, aunado a las intervenciones dietéticas como estrategia inicial de manejo en pacientes que no presenten alguna contraindicación para realizar actividad física. La determinación del tipo e intensidad de ejercicio será de manera individualizada.

El ejercicio de alta intensidad es la opción más efectiva para mejorar todos los componentes del SM, sin embargo es una actividad que no puede recomendarse a todos los individuos.

\section{DIETA MÁS EJERCICIO}

\section{Recomendación fuerte}

Se recomienda la dieta más ejercicio en los pacientes con SM para el manejo de la obesidad y sobrepeso a largo plazo. Esta estrategia conjunta es superior al ejercicio solo y con una mayor eficacia que las intervenciones dietéticas de manera aislada.

Diversos estudios marcan que la disminución de la circunferencia abdominal con pérdida de peso se asocia con mejoras significativas en los diferentes componentes del SM independientemente del sexo.
Las intervenciones conductuales son la mejor opción para prevenir el SM, en la relación riesgo-beneficio parecen más favorables que las intervenciones farmacológicas.

Se debe fijar como objetivo primario de tratamiento en los pacientes con sobrepeso/obesidad reducir el peso corporal en un 7-10\%. Como objetivo secundario lograr una normalización en las cifras de IMC $\left(I M C \leq 25 \mathrm{~kg} / \mathrm{m}^{2}\right)$.

Se recomienda como primera estrategia terapéutica para lograr estos objetivos es la dieta y ejercicio adaptado a las características individuales de cada paciente.

En aproximadamente un 40\% de los casos estas medidas provocan una reducción en la prevalencia del SM: para los adultos que son obesos (IMC 30-39.9) y tienen un alto riesgo de desarrollar $\mathrm{DM}_{2}$, recomendamos que los médicos ofrezcan intervenciones conductuales estructuradas con la finalidad de favorecer estilos de vida saludable encaminadas a la pérdida de peso.

\section{Nivel de evidencia moderada}

En una revisión sistemática se contemplaron cuatro ensayos controlados y aleatorizados, en donde se involucran la educación dietética en conjunto con el ejercicio.

En el primer estudio, la MEV se dio de acuerdo con las pautas estándar, indicando una dieta y ejercicio prescritos individualmente, sugiriendo actividad física de intensidad moderada (caminatas vigorosas durante al menos 150 minutos/semana). Los sujetos del grupo control recibieron información general enfatizando la importancia de un estilo de vida saludable por medio de sus médicos familiares, quienes otorgaban consejos de acuerdo con su práctica clínica 
habitual. En el segundo estudio, la intervención de MEV implementada fue alentar a las personas a hacer elección de un estilo de vida saludable. A los participantes en el grupo de intervención se les dio, de manera detallada e individualizada, un asesoramiento dietético y de ejercicio. Se realizaban sesiones para supervisión y se estableció un circuito de entrenamiento progresivo adaptado individualmente, en donde la resistencia a intensidad fueron moderadas y recomendadas dos veces por semana. El tercero de estos estudios fue un ensayo controlado y aleatorizado de tres grupos. En el primer grupo, los participantes recibieron un programa intensivo de MEV con los objetivos de lograr y mantener una reducción de peso de al menos un $7 \%$ de peso corporal mediante una dieta saludable baja en calorías y baja en grasas, y se les indicó que participaran en actividad física de intensidad moderada a intensa. A los participantes en el segundo grupo se les dio un estilo de vida estándar y se recomendó un placebo, y los participantes en el tercer grupo recibieron recomendaciones estándar de estilo de vida y metformina. Los pacientes recibieron semanalmente asesoramiento sobre nutrición durante las primeras cuatro semanas y posteriormente cada dos semanas. La ingesta calórica fue restringida utilizando una dieta equilibrada en el medio europeo ( $\sim 50 \%$ de hidratos de carbono, 30\% de proteína, 20 a $60 \mathrm{~g}$ de grasa/día, suplementado con vitaminas y minerales). Finalmente, en el cuarto estudio los participantes se ejercitaron de tres a cuatro veces por semana, usando un protocolo idéntico y se incluyó un programa de control de peso en pequeños grupos, de tres a cuatro miembros. El programa de control de peso fue una intervención de comportamiento basado en un manual, que se centró en cinco elementos: estilo de vida, ejercicio, actitudes, relaciones interpersonales y nutrición.

El objetivo principal de la intervención fue la pérdida de peso de 0.5 a $1 \mathrm{~kg}$ por semana, logrado gradualmente disminuyendo ingesta de calorías y grasas, y con MEV. Los objetivos dietéticos iniciales se fijaron en 1,200 kcal para mujeres y 1,500 kcal para hombres, con alrededor del 15 al 20\% de las calorías provenientes de la grasa. A los pacientes se les pidió que mantuvieran su dieta habitual y hábitos de ejercicio durante un mínimo de seis meses.

Excluyendo uno de los ocho estudios, el análisis para RR $(n=2,839)$ mostró evidencia de heterogeneidad entre los estudios ( $p<0.001$ ). El RR fue 2.0 (IC 95\%: 1.5-2.7) por el modelo de efectos aleatorios para siete intervenciones, y los otros modelos (efectos fijos y modelo bayesiano) mostraron valores similares. Cuando el análisis se repitió con los ocho estudios (incluido el estudio excluido) los resultados fueron similares. Todos los resultados indicaron que el RR fue mayor en los grupos de MEV (duración media del seguimiento, dos años) que en el grupos de control, porque solo unos pocos estudios fueron incluidos en el análisis.

La diferencia en los cambios de los diferentes componentes del SM fueron mayores en el grupo de MEV (media de un año), ya que provocó valores significativamente más reducidos (7 intervenciones, $\mathrm{n}=710$ ) en comparación con el grupo control, según el modelo de efectos aleatorios. Los valores para la glucemia en ayunas fueron de $-11.5 \mathrm{mg} / \mathrm{dl}$ (IC 95\%: -22.4 a -0.6) (5 intervenciones), $-2.7 \mathrm{~cm}$ (IC 95\%: $-4.6 \mathrm{a}-0.9)$ para la circunferencia de la cintura (6 intervenciones), -6.4 mmHg (IC 95\%: -9.7 a -3.2) para la PAS, -3.3 mmHg (IC 95\%: -5.2 a -1.4) para la PAD, y $-12.0 \mathrm{mg} / \mathrm{dl}$ (IC 95\%: -22.2 a -1.7) para los triglicéridos.

Los valores de reducción promedio no fueron significativos para HDL (1.3 mg/dl, IC 95\%: -0.6 a 3.1). Las estimaciones para los análisis de sensibilidad para $\mathrm{r}$ : 0.3 y r: 0.7 no fueron muy diferentes de la de r: 0.5 (Tabla 2). Los resultados de los análisis de subgrupos utilizando cuatro intervenciones mostraron niveles de significancia similares. 
Los principales objetivos para los sujetos con MEV intensivas eran lograr y mantener un peso reducción por medio de la dieta y el ejercicio físico de intensidad moderada. La mayoría del grupo de control recibió sugerencias estándar de MEV. Los resultados del metaanálisis de las intervenciones que involucraban ejercicio fueron estadísticamente significativos, aunque mostraron efectos pequeños tanto para los niveles de HDL como para los triglicéridos. El tamaño del efecto fue de $1.3 \mathrm{mg} / \mathrm{dl}$ (IC 95\%: -0.6 a $3.1 \mathrm{mg} / \mathrm{dl}$ ) para HDL y de $-12 \mathrm{mg} / \mathrm{dl}$ (IC 95\%: -22 a -2 mg/dl) para los triglicéridos por el modelo de efectos aleatorios, y de $2.9 \mathrm{mg} / \mathrm{dl}$ (IC 95\%: 2.5-3.3 mg/dl) y -19 mg/dl (IC 95\%: -20 a -17 mg/dl), respectivamente, por el modelo de efectos fijos. Para este estudio las MEV incluían la dieta sola o dieta más ejercicio dentro del mismo concepto.

En otra revisión sistemática, la cual incluyó estudios con mayor tiempo de seguimiento, se comparó la eficacia a largo plazo de la dieta más ejercicio vs. solo dieta, dieta más ejercicio vs. solo ejercicio y dieta vs. ejercicio. Se incluyeron 21 estudios que involucraron 3,521 participantes. Cuando se comparó la dieta y la dieta más ejercicio, esta última produjo una reducción significativamente más pronunciada en el peso corporal (diferencias de medias [DM]: $-1.38 \mathrm{~kg}$; IC 95\%: -1.98 a -0.79], y masa grasa (DM: - 1.65 kg; IC 95\%: -2.81 a -0.49], respectivamente. Al comparar la dieta más ejercicio vs. solo ejercicio, la desviación media en el cambio de peso corporal (-4.13 kg; IC 95\%: -5.62 a -2.64), circunferencia de cintura $(-3.00 \mathrm{~cm}$; IC 95\%: -5.81 a -0.20$)$ y la masa grasa ( $-3.60 \mathrm{~kg}$; IC 95\%: $-6.15 \mathrm{a}-1.05$ ) estuvieron a favor de la dieta combinada con ejercicio. Al comparar ejercicio vs. solo dieta, la dieta resultó en una disminución más pronunciada del peso corporal (DM: $-2.93 \mathrm{~kg}$; IC 95\%: -4.18 a -1.68) y masa grasa (DM: -2.20 kg; IC 95\%: -3.75 a -0.66). La dieta más ejercicio también proporcionó la mayor reducción en los lípidos en sangre y la presión arterial en comparación con dieta y ejercicio de manera individual. Los resultados de los metaanálisis de la red confirmaron estos hallazgos.

La AHA y la ADA han alertado de los probables riesgos para la salud tras el uso de edulcorantes artificiales en lugar de azúcar para combatir la obesidad, el SM y la diabetes, aunque aún hay incertidumbre sobre los daños a la salud de las bebidas endulzadas artificialmente. Varios estudios observacionales de gran tamaño, como el estudio sobre el riesgo de aterosclerosis en comunidades, el estudio del corazón de Framingham y el estudio multiétnico de la aterosclerosis informaron una asociación positiva entre el consumo de refrescos de dieta y el aumento de los riesgos del SM y la DM2.

\section{Estrategias de tratamiento farmacológico con mayor eficacia para el SM en el primer nivel en los adultos ${ }^{31-35}$ (Tablas 5 y 6)}

\section{Punto de buena práctica}

En las personas para quienes la MEV no es suficiente y se considera que tienen un alto riesgo de enfermedad cardiovascular, se debe evaluar la necesidad de utilizar una terapia farmacológica para tratar los componentes individuales del SM.

\section{Punto de buena práctica}

No existen metas de control para definir cuál es el nivel adecuado de cada uno de los factores que integran el SM, sin embargo se pueden aplicar los criterios de metas y tratamiento considerando en forma individual cada componente, sobrepeso y obesidad, hiperglucemia intermedia y diabetes, hipertensión arterial y dislipidemia. 
TABLA 5. ¿Cuál es el manejo con mayor eficacia para el tratamiento del SM en adultos?

P: población adulta con SM; I: modificaciones en el estilo de vida, tratamiento farmacológico, modificaciones al estilo de vida + tratamiento farmacológico; C: tratamiento farmacológico, modificaciones en el estilo de vida, control, ejercicio, ejercicio + modificaciones en el estilo de vida, medicamentos antidiabéticos, dieta, dieta + ejercicio, medicamentos antiobesidad, medicamentos antiobesidad + modificaciones en el estilo de vida, ejercicio; O: peso, IMC, circunferencia abdominal, colesterol total, colesterol HDL, glucosa en ayuno, presión arterial sistólica, presión arterial diastólica.

\begin{tabular}{|c|c|c|c|c|c|}
\hline \multirow[b]{2}{*}{ Comparación } & \multicolumn{3}{|c|}{ Metaanálisis directo } & \multicolumn{2}{|c|}{ Metaanálisis de comparaciones mixtas } \\
\hline & n. ${ }^{\circ}$ de estudios & OR (IC 95\%) & $I^{2}$ & $\mathrm{n} .^{\circ}$ de comparaciones & OR (IC 95\%) \\
\hline \multicolumn{6}{|l|}{ Red agrupada } \\
\hline $\begin{array}{c}\text { Estilo de vida } \\
\text { vs. control }\end{array}$ & 8 & $3.81(2.47-5.88)$ & 56.8 & 8 & $4.43(2.36-9.16)$ \\
\hline $\begin{array}{l}\text { Tratamiento } \\
\text { farmacológico } \\
\text { vs. control }\end{array}$ & 5 & $1.59(1.04-2.45)$ & 69.1 & 5 & $1.73(0.73-3.809$ \\
\hline $\begin{array}{l}\text { Estilo de vida } \\
\text { y tratamiento } \\
\text { farmacológico } \\
\text { vs. control }\end{array}$ & 1 & $1.14(0.20-1.24)$ & - & 1 & $2.16(0.63-8.29)$ \\
\hline $\begin{array}{l}\text { Tratamiento } \\
\text { farmacológico } \\
\text { vs. estilo de vida }\end{array}$ & 3 & $0.49(0.20-1.24)$ & 80.8 & 3 & $0.39(0.16-0.90)$ \\
\hline $\begin{array}{l}\text { Estilo de vida } \\
\text { y tratamiento } \\
\text { farmacológico } \\
\text { vs. estilo de vida }\end{array}$ & 2 & $0.53(0.26-10.06)$ & 0 & 2 & $0.49(0.14-1.74)$ \\
\hline $\begin{array}{l}\text { Estilo de vida } \\
\text { y tratamiento } \\
\text { farmacológico } \\
\text { vs. tratamiento } \\
\text { farmacológico }\end{array}$ & 2 & $1.31(0.11-15.13)$ & 91.9 & 2 & $1.25(0.36-4.49)$ \\
\hline
\end{tabular}

\section{Red completa}

\begin{tabular}{|c|c|c|c|c|c|}
\hline Control & & Referencia & & \multicolumn{2}{|c|}{ Referencia } \\
\hline $\begin{array}{l}\text { Medicamentos } \\
\text { antidiabéticos }\end{array}$ & 3 & $2.56(1.07-6.13)$ & 78.4 & - & $3.63(1.28-11.91)$ \\
\hline $\begin{array}{l}\text { Medicamentos } \\
\text { antidiabéticos } \\
\text { y estilo de vida }\end{array}$ & 1 & $1.14(0.48-2.729$ & - & - & $1.74(0.31-10.47)$ \\
\hline $\begin{array}{r}\text { Medicamentos } \\
\text { antiobesidad }\end{array}$ & 1 & $1.50(0.99-2.29)$ & - & - & $1.20(0.27-5.39)$ \\
\hline $\begin{array}{l}\text { Medicamentos } \\
\text { antiobesidad } \\
\text { y estilo de vida }\end{array}$ & 0 & - & - & - & $5.10(0.59-47.13)$ \\
\hline Dieta & 2 & $7.07(3.84-13.0)$ & 0 & - & $4.64(1.25-18.52)$ \\
\hline Ejercicio & 3 & $2.22(1.03-4.78)$ & 0 & - & $2.05(0.62-7.27)$ \\
\hline Ejercicio y dieta & 5 & $4.08(2.33-7.16)$ & 21.1 & - & $6.73(3.05-17.95)$ \\
\hline
\end{tabular}

SM: síndrome metabólico; IMC: índice de masa corporal; HDL: high densitiy lipoproteins; IC: intervalo de confianza; OR: razón de momios. Adaptada de Dunkley, et al., 201227. 
TABLA 6. ¿Cuál es la eficacia del manejo conductual en comparación con el manejo farmacológico sobre los componentes del SM en adultos?

\begin{tabular}{|c|c|c|c|c|}
\hline $\begin{array}{l}\text { P: población adulta con SN } \\
\text { colesterol HDL, glucosa en } \\
\text { Efecto de los tratamientos }\end{array}$ & $\begin{array}{l}\text { onductı } \\
\text { ón arte } \\
\text { jetivos }\end{array}$ & $\begin{array}{l}\text { macológico; 0: } p \\
\text { ión arterial diast } \\
\text { adarios del síndro }\end{array}$ & erenci & esterol total, \\
\hline Resultado & & Tipo & & \\
\hline & & Diferencia & $5 \%)$ & \\
\hline & & & & gico \\
\hline Primario & & & & \\
\hline Peso (kg) & -3.13 & $(-3.88 a-2.38)$ & -2.89 & $(-3.49 a-2.29)$ \\
\hline IMC & -1.09 & $(-1.43 a-0.75)$ & -1.27 & $(-1.82 \mathrm{a}-0.72)$ \\
\hline Circunferencia abdominal & -3.05 & $(-3.86 a-2.24)$ & -2.29 & $(-3.04 a-1.55)$ \\
\hline Secundarios & & & & \\
\hline Colesterol total, mmol/l & -0.10 & $(-0.18 a-0.03)$ & -0.33 & $(-0.42 a-0.24)$ \\
\hline Colesterol LDL, mmol/I & -0.14 & $(-0.29 a-0.00)$ & -0.28 & $(-0.38 a-0.19)$ \\
\hline Glucosa en ayuno, $\mathrm{mmol} / \mathrm{l}$ & -0.14 & $(-0.23 a-0.05)$ & -0.43 & $(-0.66 a-0.20)$ \\
\hline $\begin{array}{l}\text { Presión arterial sistólica, } \\
\mathrm{mmHg}\end{array}$ & -1.76 & $(-2.61 \mathrm{a}-0.91)$ & -1.70 & $(-2.28 \mathrm{a}-1.13)$ \\
\hline $\begin{array}{l}\text { Presión arterial diastólica, } \\
\mathrm{mmHg}\end{array}$ & -1.60 & $(-2.27$ a -0.93$)$ & -1.24 & $(-1.88 a-0.61)$ \\
\hline
\end{tabular}

IMC: índice de masa corporal; IC: intervalo de confianza; LDL: low-density lipoprotein; SM: síndrome metabólico; HDL: high densitiy lipoproteins.

Adaptada de Dunkley. et al., $2012^{27}$.

\section{Punto de buena práctica}

No existe evidencia o es de baja calidad para identificar un tratamiento farmacológico que sea específico para SM, la evidencia que existe está con base en el tratamiento para cada uno de los componentes individuales de este, por lo que se sugiere que se apliquen las guías específicas a cada uno de ellos.

\section{Recomendación fuerte}

En los pacientes con SM, la alteración en la glucemia debe ser tratada conforme a la presencia de hiperglucemia intermedia o diabetes de acuerdo con las guías actuales, siendo el medicamento inicial sugerido la metformina, aunado a MEV.

\section{Recomendación fuerte}

Se deber realizar la determinación del mejor enfoque farmacológico para manejar la dislipidemia para pacientes con SM dependiendo del riesgo conocido o estimado de enfermedad cardiovascular ateroesclerótica, que puede variar ampliamente entre los pacientes que cumplen con los criterios para el SM. El objetivo primario deberá ser reducir el colesterol LDL y posteriormente el colesterol no HDL.

\section{Recomendación fuerte}

La presión arterial objetivo para pacientes con cardiopatía coronaria, diabetes o enfermedad renal crónica es < 130/80 mmHg, mientras que para pacientes sin estas comorbilidades es < 140/90 mmHg. 
Si estos objetivos no se pueden lograr con la MEV, se requiere medicación antihipertensiva. Se recomienda utilizar un inhibidor de la enzima convertidora de angiotensina (IECA) si las cifras de presión arterial son $\geq 140 / 90 \mathrm{mmHg}$ en los pacientes con SM y como opción un antagonista de los receptores de angiotensina II.

\section{CONCLUSIONES}

Existen múltiples limitaciones para establecer una estrategia de manejo integral del SM basada en evidencia robusta y de adecuada calidad, ya que no existen documentos de tipo prospectivo que incluyan ensayos controlados y aleatorizados, revisiones sistemáticas con metaanálisis, con intervenciones dirigidas exclusivamente a revertir o prevenir el SM. Por tanto existe un vacío importante de información que limita la generación de una GPC elaborada con metodología GRADE. Los métodos utilizados para la revisión de la literatura en la presenta guía fueron robustos, a pesar de la poca información útil disponible.

Queda de manifiesto que las diferencias observadas al utilizar los diversos criterios de diagnóstico, reflejan diferentes puntos de vista de los autores sobre la etiología y las bases fisiopatológicas del SM.

Hubo considerable heterogeneidad clínica entre las intervenciones incluidas en las revisiones sistemáticas utilizadas. Adicionalmente, existen diferencias entre las poblaciones de estudio, el tiempo de seguimiento y el tratamiento de los sujetos del grupo de control, lo cual dificulta las comparaciones directas.

Aunado a lo anterior, en la revisión de los resultados de los metaanálisis se demuestra que la reversión del SM podría representar desde una mejoría significativa de varias anomalías metabólicas hasta solo una leve mejora de un componente del síndrome, en algunos casos, el uso de puntos de corte de valores dicotómicos en lugar de la utilización de valores continuos para los componentes individuales no permite considerar las modificaciones en la magnitud de los efectos.

A pesar de todas las limitantes mencionadas, la tendencia de la eficacia en el manejo del SM se centra en llevar estilos de vida saludable que ayuden a prevenir o retardar las complicaciones cardiovasculares y el desarrollo de DM2. Las recomendaciones presentadas en esta guía deben ayudar a los médicos y pacientes a implementar y comprender las intervenciones más apropiadas para prevenir y tratar el SM en el primer nivel de atención.

\section{AGRADECIMIENTOS}

Agradecemos el apoyo recibido por el Licenciado en Bibliotecología Enrique Juárez Sánchez, de la Facultad de Filosofía y Letras de la Universidad Nacional Autónoma de México, para la realización de la búsqueda de la información.

\section{FINANCIAMIENTO}

No se recibió financiamiento alguno para el desarrollo de esta guía.

\section{CONFLICTO DE INTERESES}

Los autores no tienen conflictos de interés que declarar.

\section{BIBLIOGRAFÍA}

1. Grundy SM. Metabolic syndrome update. Trends Cardiovasc Med. 2016;26(4):364-73. 
2. O'Neill S, O'Driscoll L. Metabolic syndrome: a closer look at the growing epidemic and its associated pathologies. Obes Rev. 2015;16(1):1-12.

3. Kaur J. A comprehensive review on metabolic syndrome. Cardiol Res Pract. 2014;2014:943162.

4. Cuevas A, Alvarez V, Carrasco F. Epidemic of metabolic syndrome in Latin America. Current Opinion Endocrinol Diab Obes. 2011;18:134-8.

5. Escobedo J, Schargrodsky H, Champagne B, Silva H, Boissonet CP, Vinueza R, et al. Prevalence of the metabolic syndrome in Latin America and its association with sub-clinical carotid atherosclerosis: the CARMELA cross sectional study. Cardiovasc Diabetol. 2009;8: 52-61.

6. De Carvalho Vidigal F, Bressan J, Babio N, Salas-Salvadó J. Prevalence of metabolic syndrome in Brazilian adults: a systematic review. BMC Public Health. 2013;13:1198.

7. Márquez-Sandoval F, Macedo-Ojeda G, Viramontes-Homer D, Fernández Ballart JD, Salas-Salvadó J, Vizamos B. The prevalence of metabolic syndrome in Latin America: a systematic review. Public Health Nutrition. 2011;14:1702-13.

8. Edwardson CL, Gorely T, Davies MJ, Gray LJ, Khunti K, Wilmot EG, et al. Association of sedentary behaviour with metabolic syndrome: a meta-analysis. PLoS One. 2012;7(4):e34916.

9. Rodríguez-Monforte M, Sánchez E, Barrio F, Costa B, Flores-Mateo G. Metabolic syndrome and dietary patterns: a systematic review and meta-analysis of observational studies. Eur J Nutr. 2017;56:925-47.

10. Rothberg AE, McEwen LN, Kraftson AT, Ajluni N, Fowler CE, Nay CK, et al. Impact of weight loss on waist circumference and the components of the metabolic syndrome. BMJ Open Diabetes Res Care. 2017;20;5(1):e000341.

11. Lloyd LJ, Langley-Evans SC, McMullen S. Childhood obesity and risk of the adult metabolic syndrome: a systematic review. Int J Obes. 2012;36:1-11.

12. Kim J, Lee I, Lim S. Overweight or obesity in children aged 0 a 6 and the risk of adult metabolic syndrome: A systematic review and meta-analysis. J Clin Nurs. 2017;26:3869-80.

13. Meigs JB, Wilson PW, Fox CS, Vasan RS, Nathan DM, Sullivan LM, et al. Body mass index, metabolic syndrome, and risk of type 2 diabetes or cardiovascular disease. J Clin Endocrinol Metab. 2006;91(8): 2906-12.

14. Schellong K, Schulz S, Harder T, Plagemann A. Birth weight and longterm overweight risk: systematic review and a meta-analysis including 643,902 persons from 66 studies and 26 countries Globally. PLoS One. 2012;7(10):e47776.

15. Lipińska A, Koczaj-Bremer M, Jankowski K, Kaźmierczak A, Ciurzyński M, Ou-Pokrzewińska A, et al. Does family history of metabolic syndrome affect the metabolic profile phenotype in young healthy individuals? Diabetol Metab Syndr. 2014;6:75.

16. Hu T, Mills KT, Yao L, Demanelis K, Eloustaz M, Yancy WS Jr, et al. Effects of low-carbohydrate diets versus low-fat diets on metabolic risk factors: a meta-analysis of randomized controlled clinical trials. Am J Epidemiol. 2012;176 Suppl 7:S44-54.

17. Pattyn N, Cornelissen V, Toghi ES, Vanhees L. The effect of exercise on the cardiovascular risk factors constituting the metabolic syndrome. Sports Med. 2013;43:121-33.

18. Xu Y, Shen S, Sun L, Yang H, Jin B, Cao X. Metabolic syndrome risk after gestational diabetes: a systematic review and meta-analysis. PLoS One. 2014;9(1):e87863.

19. Vancampfort D, Stubbs B, Mitchell AJ, De Hert M, Wampers M, Ward PB, et al. Risk of metabolic syndrome and its components in people with schizophrenia and related psychotic disorders, bipolar disorder and major depressive disorder: a systematic review and meta-analysis. World Psychiatry. 2015;14:339-47.
20. Behboudi GS, Amiri M, Bidhendi YR, Noroozzadeh M, Farahmand M, Rostami DM, et al. The risk of metabolic syndrome in polycystic ovary syndrome: a systematic review and meta-analysis. Clin Endocrino (Oxf). 2018;88(2):169-84.

21. Onesi SO, Ignatius UE. Metabolic syndrome: Performance of five different diagnostic criterias. Indian J Endocrinol Metab. 2014;18(4): 496-501.

22. Koponen HJ, Hakko HH, Saari KM, Lindeman SM, Karvonen KM, Isohanni MK, et al. The prevalence and predictive value of individua criteria for metabolic syndrome in schizophrenia: a Northern Finland 1966 Birth Cohort Study. World J Biol Psychiatry. 2010;11(2):262-7.

23. Strazzullo P, Barbato A, Siani A, Cappuccio FP, Versiero M, Schiattarella $\mathrm{P}$, et al. Diagnostic criteria for metabolic syndrome: a comparative analysis in an unselected sample of adult male population. Metabolism. 2008; 57:355-61.

24. Rothberg AE, McEwen LN, Kraftson AT, Ajluni N, Fowler CE, Nay CK, et al. Impact of weight loss on waist circumference and the components of the metabolic syndrome. BMJ Open Diabetes Res Care. 2017;5(1):e000341.

25. Yamaoka K, Tango T. Effects of lifestyle modification on metabolic syndrome: a systematic review and meta-analysis. BMC Med. 2012; 10:138.

26. Leão LS1, de Moraes MM, de Carvalho GX, Koifman RJ. Nutritiona interventions in metabolic syndrome: a systematic review. Arq Bras Cardiol. 2011;97(3):260-5.

27. Dunkley Aj, Bodicoat DH, Greaves CJ, Russell C, Yates T, Dvies MJ, et al. Diabetes prevention in the real world: effectiveness of pragmatic lifestyle interventions for the prevention of type 2 diabetes and of the impact of adherence a guideline recommendations: a systematic review and meta-analysis. Diabetes Care. 2014;37(4):922-33.

28. Hu T, Mills KT, Yao L, Demanelis K, Eloustaz M, Yancy WS Jr, et al. Effects of low-carbohydrate diets versus low-fat diets on metabolic risk factors: a meta-analysis of randomized controlled clinical trials. Am J Epidemiol. 2012;176 Suppl 7:S44-54.

29. Peirson L, Douketis J, Ciliska D, Fitzpatrick-Lewis D, Ali MU, Raina P. Treatment for overweight and obesity in adult populations: a systematic review and meta-analysis. CMAJ Open. 2014;2(4):E306-17.

30. Schwingshackl L, Dias S, Hoffmann G. Impact of long-term lifestyle programs on weight loss and cardiovascular risk factors in overweight/obese participants: a systematic review and network meta-analysis. Syst Rev. 2014;3:130.

31. Grundy SM, Stone NJ, Bailey AL, Beam C, Birtcher KK, Blumenthal RS et al. 2018 AHA/ACC/AACVPR/AAPA/ABC/ACPM/ADA/AGS/APhA/ASPC/ NLA/PCNA Guideline on the Management of Blood Cholesterol: A Report of the American College of Cardiology/American Heart Association Task Force on Clinical Practice Guidelines. J Am Coll Cardiol. 2019;73(24):3168-209.

32. Metabolic syndrome [Internet]. BMJ best practice; 2018. Disponible en: https://bestpractice.bmj.com/topics/en-gb/212/pdf/212.pdf

33. Brauer P, Connor S, Shaw E, Singh H, Bell N, Shane AR, et al. Canadi an Task Force on Preventive Health Care. Recommendations for prevention of weight gain and use of behavioural and pharmacologic interventions a manage overweight and obesity in adults in primary care. CMAJ. 2015;187(3):184-95.

34. Hoare E, Varsamis P, Owen N, Dunstan DW, Jennings GL, Kingwell BA. Sugar- and intense-sweetened drinks in Australia: A systematic review on cardiometabolic risk. Nutrients. 2017;9(10).

35. Azad MB, Abou-Setta AM, Chauhan BF, Rabbani R, Lys J, Copstein L, et al. Nonnutritive sweeteners and cardiometabolic health: a systematic review and meta-analysis of randomized controlled trials and prospective cohort studies. CMAJ. 2017;189(28):E929-39. 